\title{
A Study on The Relationship Between Burnout and Quality of Life Among Nurses In Thu Duc District Hospital, Vietnam
}

\section{Minh Quan Nguyen}

Thu Duc District hospital

Phuong Anh Hoang Le

Thu Duc District hospital

Minh Hoang Nguyen Vo ( $\square$ minhhoangytcc87@gmail.com )

Thu Duc District hospital https://orcid.org/0000-0001-5657-8272

\section{Research}

Keywords: Nurses, Burnout, Quality of life, Staff healthcare

Posted Date: June 11th, 2020

DOI: https://doi.org/10.21203/rs.3.rs-33664/v1

License: (c) (i) This work is licensed under a Creative Commons Attribution 4.0 International License.

Read Full License 


\section{Abstract}

Purpose: The purpose of this study was to investigate the levels of the burnout among nurses, and to further explore the effects of the burnout on their quality of life.

Methods: This study is a cross-sectional design. 280 nurses of Thu Duc District hospital in Vietnam were interviewed with structured questionnaires. Questionnaires included "Demographic Inventory", "MBI scale", and "SF-36 Scale". T-test, ANOVA, Pearson's correlation, and Regression Analysis were used to analyze data.

Results: (1). The nurses had low and middle levels of the burnout, and moderate levels of the quality of life. (2) There were significant correlations between demography and burnout, including gender, education, department, main work shift, and years in this department. (3) There is a significant negative relationship in many sub-scales of the burnout and quality of life scale. (4) Education and burnout predicted $17.6 \%$ of the variance in quality of life.

Conclusion: There were clear evidences that the nurses experienced burnout and then their quality of life was negatively affected. It is suggested that programs must be created to prevent burnout in nurses.

\section{Introductions}

According to the Global Health Observatory [1], about 48\% of WHO Member States have less than 3 nursing and midwifery personnel per 1000 population (about $27 \%$ report to have less than 1 ). Although nursing is a highly demanding profession in society, it plays a central role in the provision of health care in all countries, including in developed or developing countries [2]. However, this is a serious shortage of human resources. A nurse has to care for many patients. This has led to occupational stress of nurses. Several types of occupational stress have been identified, including burnout, compassion fatigue, vicarious traumatization [3]. This is the reason why many nurses have left the profession.

In 1975, for the first time, the term burnout was used by Herbert Freudenberger to describe this situation of health care workers. It is physical, emotional and mental exhaustion due to long-term engagement in work situations that requires affection $[2,4]$. Previous studies have found a significant relationship between burnout and quality of life among nurses. Almost burnout and quality of life are negatively correlated, increased burnout leads to lower quality of life [2, 5-7]. In summary, measuring burnout among nurses is important because their well-being has implications for stability in the healthcare workforce and the quality of care provided [4].

According to the Global Health Observatory [1], in Vietnam, the proportion of nurses per 1,000 people is about 1.2. Each nurse must care for more than 40 patients each day [8]. A survey by the Institute of Occupational Hygiene and national shows, at the Emergency Department, around $23 \%$ of employees with high stress, $42 \%$ of them had moderate stress [9]. Therefore, to complete the task, nurses not only face pressure from the patient but also face other problems relationships, work environment and income. 
However, in Vietnam, the concept of burnout has been ignored, no research has addressed burnout. This study provide useful information, forecasting the burnout of nurses in Vietnam. Results of this research will help nurses to identify their burnout and improve their quality of life.

\section{Purpose}

The general purpose of this research aimed to investigate the level of burnout and the quality of life of nurses. Specific objectives included as below:

(1) to explore what demographic characteristics, levels of burnout and the quality of life among nurse,

(2) to examine relationships between among demographic characteristics, burnout and the quality of life among nurse, and

(3) to investigate the significant predictors of the quality of life among nurse

\section{Materials And Methods}

\section{Research design:}

This study is a cross-sectional, descriptive, and correlational design.

\section{Research Setting}

The survey was conducted at Thu Duc District hospital. Thu Duc District hospital is a public general hospital in North East of Ho Chi Minh City. This hospital has 800 beds, dedicated to serving the local people. Most people come for medical examination and treatment with the support of universal health insurance. Every day around 4000 people visit the clinic. According to personnel statistics at Thu Duc Hospital in 2016, the total number of nurses is about 590 . The ratio of nurses/beds is $1 / 1.2$. This ratio is lower than the national average of $1.2 / 1$ to $1.5 / 1$ [8].

\section{Research participants:}

Official nurses were recruited in the study. They were ready to participate in the study and signed in the consent form. These people were enrolled in the study. Absent nurses were excluded from the study. Absences include postpartum leave, post-holiday leave, unpaid leave, sick leave, business trip, etc.

\section{Sample size:}

Stratified random sampling was used throughout the study period. In Krejcie and Morgan [10] at N =600, the sample size representative of the nurses in this case was 234. The actual sample size was 280 (add $20 \%$ to prevent sample loss).

\section{Research instruments:}


A structured questionnaire, including "Demographic Inventory", "MBI scale", and "SF-36 Scale", in an interview format was conducted to collect data.

\section{Validity and reliability}

To check the validity of the questionnaire, the researcher consulted five experts. The result was acceptable because authors have shown that S-CVI of 0.80 or higher was acceptable $[11,12]$.

The results of pilot test showed that the reliability of the questionnaire was acceptable with Cronbach's Alpha of the MBI questionnaire of 0.824 and Cronbach's Alpha of the SF-36 questionnaire of 0.865 .

\section{Data collection process}

Thirty nurses were recruited in a pilot study with the aim of clarifying the questionnaire and testing the reliability of the questionnaire as well. Participants were sent an email about the appointment with the researcher. Each participant was completed the interview for 30 minutes.

The researcher in this study instructed the participants how to complete the questionnaire and explain the variables. They did not present any personal comments (for example, suggesting answers, expressing emotions, answering right or wrong, etc.) in order to limit the bias caused by the researcher. Each individual participant was surveyed separately.

\section{Data analysis}

Data was analyzed using SPSS for Windows version 22.0. T-test, ANOVA, Pearson correlation, Scheffe's method, stepwise regression model was used for theoretical analysis, explaining the hypothesis of the study.

\section{Results}

\section{Demographic characteristics}

Demographic characteristics are presented through 13 factors, 280 valid responses have been accepted. Nurses participating in the study were young. From twenty one to thirty years old with 210 cases accounted for the highest rate of $75 \%$. Female occupied the majority. Female/male ratio was 3.06 with $75.4 \%$ female and $24.6 \%$ male. The majority of participants were non-religious with 163 cases (58.2\%). Singles and marriages accounted for almost the same proportion and dominate. Most of participants did not have children. This rate was high 162 (57.9\%). Intermediate degree nurses accounted for the majority, with 192 cases $(68.6 \%)$. One hundred eighty-nine cases were not the main source of family income (67.5\%). Lower middle income (> 24-94 million VND / year) account for most of 217 cases (77.5\%).

Because this study uses stratified sampling, the percentage of departments was predicted before the survey. The most common were Wards with 129 cases (46.1\%). The majority of respondents were nurses with 256 cases (91.4\%). Most nurses worked 8-hours shifts with 143 cases (51.1\%). Among the nurses 
interviewed, most people who worked from 1-3 years (29.6\%). Similarly, when surveying years in this department, I have found that nurses working in this department for 1-3 years were the majority (33.6\%).

\section{Burnout among nurse (Table 1)}

\section{The quality of life among nurse (Table 2)}

\section{Relationships between demographic characteristics and burnout}

College level had a lower level of burnout than other groups $(F=9.005, p<0.001)$. Nurses working in Wards and operating rooms (OR) had significantly higher levels of burnout than Outpatient department (OPD) with $F=4.009, p<0.001$. For main work shift, the results showed that $8 \mathrm{~h}$ shifts and administrative nurses had a significantly higher burnout score than $24 \mathrm{~h}$ shifts $(F=4.821, p<0.01)$. Years in nursing had a relationship with burnout, but the Scheffé test did not show any specific differences for each pair $(F=$ $2.419, p<0.05)$. For years in this department, 7-9 years nurses group, who had higher levels of burnout than 1 year and below group $(F=3.201, p<0.05)$.

\section{Relationships between demographic characteristics and the quality of life among nurse}

Mean score the quality of life in the nursing college level was significantly higher than intermediate $(\mathrm{F}=$ $8.453, p<0.001)$. Nurses working in the outpatient department and other department had a significantly higher quality of life in operating room $(F=4.376, p<0.01)$. For years in nursing, nurses working less than 1 year had a significantly higher quality of life compared to $4-6$ years and 7-9 years $(F=4.360, p<0.01)$. For Years in this department, nurses working less than 1 year had a significantly higher quality of life compared to other groups $(F=7.799, p<0.001)$.

\section{Relationships between burnout and the quality of life among nurse (Table 3)}

\section{The significant predictors of the quality of life among nurse (Table 4)}

\section{Discussions}

The majority of nurses participating in the study were young. Female was majority in this study. This is quite understandable because many nursing studies also show that women dominate this profession [4, $13,14]$. Non-religious nurses made up the majority of this study. For education, Intermediate degree nurses accounted for the majority. This result is consistent with cultural and religious characteristics in Vietnam. The frequency of singles and marriages was almost the same and predominates. Most of the subjects did not have children. Because the percentage of singles was the highest, this result was appropriate. Most of the participants were not the main source of family income. The nurses' age was so young that most of them were not the main source of family income. Comparing the results of this study and other studies in the World, I find that nurses in Vietnam had a much lower income than many other countries. Most nurses in this study work with 8 hours shift. In Vietnam, the nurses work 8-hour shifts, which means there were 4 groups and they had continuously rotating shift. Among the nurses 
interviewed, most nurses had little experience. Age of the study participants was so young that this result is appropriate.

The mean scores of overall burnout were $2.38 \pm 0.75$ (range from 0.40 to 4.55 ). The burnout among nurses was below average, even for internal factors (Emotional Exhaustion, Depersonalization and Personal Accomplishment). These results were lower than most other studies in the world. According to a study by Aytekin, Yilmaz, Kuguoglu ${ }^{15}$, the author examined 80 nurses, the results showed that nurses undergoing moderate burnout on Emotional Exhaustion and Personal Accomplishment. Or Kapucu, Akkuş, Akdemir, Karacan ${ }^{16}$ found that Hemodialysis nurses had high scores for Emotional Exhaustion. In an international study on hospital care, Aiken and colleagues demonstrated that nurses were burnout at significantly higher rates than expected for health workers based on national standards [17]. Almost all newspapers in Vietnam mentioned the lack of serious human resources in nursing professional. At the hospital I surveyed also have a similar situation. Nurses often complain that they were exhausted and stressed in their work. However, the results in this study show that burnout levels were better than expected. In addition, health managers also need to consider the causes and factors that have caused burnout. Because I recognized that there were high score of participants. On the other hand, I also need to consider whether there exists nurses experienced burnout high, did not adapt, did not respond to the stress of the job and they had left their job. This finding suggested that although burnout levels were different among nurse, in general, burnout experiences in all three areas were low and relatively positive. However, the MBI score was not as predictable as mentioned above. It was only useful to compare the burnout levels between objects in the same context. However, I had difficulty in considering burnout in the general context of nurses in Vietnam because there is almost no research in Vietnam had addressed this topic. This was also a limitation of the research that need to be addressed and addressed in future studies.

The results of this study indicated that most of the nurses had moderate quality of life. Similarly, other studies had also shown that the quality of life of nurses was moderate [18-20]. The quality of life of health care workers was a topic that many scholars in the world were concerned about. The standard of quality of life includes not only income, wealth and employment, but also the social environment, environment, health (physical) and spiritual, educational, entertainment and private life [21]. As Milosevic, Golubic, Knezevic, Golubic, Bubas, Mustajbegovic ${ }^{22}$ said that quality of healthcare depends on many factors, including health, quality of life and work ability of healthcare workers. The physical and mental health of the participants are as good as the higher the score. Physical health includes Physical activity (PF), Role limitations due to physical health (RP), Pain (BP), General health (GH). Mental health includes Role Limitations Due to emotional problems (RE), Energy/ fatigue (EF), Emotional well-being (EW), Social functioning (SF). The results of this study showed that physical and mental health were above average. This result is positive and relatively uniform across regions, this is different from other such studies $[7,23,24]$.

The purpose of this study was to examine the relationship between burnout and quality of life. This result proved one of the original assumptions. There was clear evidence that the extent of burnout has a negative impact on quality of life in nursing professionals. Emotional Exhaustion, Depersonalization 
appears to be correlated negatively with most of item of QOL. Diminished Personal Accomplishment was negatively correlated with physical functioning, role emotional limitations due to health, emotional wellbeing. This result was similar to many studies around the world. Burnout was proven by many studies that had negative impacts on human health. Elevated rates of general physical illness appear to be more common in people experiencing higher degrees of burnout compared to people with lower degrees of burnout [25]. A longitudinal study of 406 social workers, found that employees with higher initial levels of burnout ultimately had more health complaints later in the study. Thus, the findings of this study fit in with the majority of burnout studies [26].

According to multiple regression analysis, the results showed that there were two variables of education and burnout that had statistically significant effects on the dependent variable. This means that, many factors in this study were found to be related to QOL. However, only education and burnout had significant levels of influence, they contributed to the change of the QOL of nurses. The results clearly showed that burnout had more influence than other variables. Research predicts that in the condition that the other variables did not change, when Burnout increases one unit, the quality of life decreases by 0.339. Education and burnout predicted $17.6 \%$ of the variance in quality of life.

\section{Conclusions}

Nurses had low and middle levels of the burnout. Quality of life among nurses was moderate. There is a significant negative relationship in many sub-scales of the burnout and quality of life scale. Education and burnout predicted $17.6 \%$ of the variance in quality of life. In clinical practice, health managers need to support nurses in improving their education and improving burnout to promote quality of life among nurses.

\section{Declarations}

\section{ACKNOWLEDGMENTS}

I would like to send a special thank you to Dr. Ya-Fen Lien, who has spent a lot of time and patience to guide me through this study. In addition, I would like to thank all the professors of the Nursing Department at Meiho University for facilitating, providing timely support and providing valuable information to me. I would also like to thank the Thu Duc district hospital, colleagues who helped me to approach and interview all participants.

\section{FUNDING}

This research is self-funding by the authors.

\section{CONTRIBUTIONS}


NQ and HA designed the study. NQ and HA collected the data. NQ and HA analysed the data. NQ, HA and $\mathrm{NH}$ contributed to the interpretation of results. All authors have read and approved the final version of the manuscript.

\section{ETHICS DECLARATIONS}

\section{Conflict of interest}

All authors declare they have no financial or non-financial interests that may be relevant to the submitted work.

\section{Ethical approval}

Approval for this study was obtained from the Thu Duc District Hopsital Science Research Ethics Board. To participants, the researcher informed nurses that they have been enrolled in the study and described the purposes of the study. The nurses voluntarily agreed to participate in by signing on informed consent. If the participant did not agree, they would be excluded from the study. Additionally, all information related to participants was solely used in the present study, not for other purposes.

After collecting, the survey samples were filled in ID. At the end of the survey, the questionnaire was surveyed and the study consent form was stored separately to avoid disclosing the participant's personal information.

\section{References}

1. Global Health Observatory. Density of nursing and midwifery personnel (total number per 1000 population, latest available year). 2013.

2. Fradelos $E$, Mpelegrinos S, Mparo C, et al. Burnout syndrome impacts on quality of life in nursing professionals: The contribution of perceived social support. Progress in Health Sciences 2014;4(1): 102.

3. Sabo B. Reflecting on the concept of compassion fatigue. Online Journal of Issues in Nursing 2011;16(1).

4. Naz S, Hashmi AM, Asif A. Burnout and quality of life in nurses of a tertiary care hospital in Pakistan. JPMA The Journal of the Pakistan Medical Association 2016;66(5): 532-36.

5. Bozorghi NHA, Nikbakhsh R, Tojari F. The relationship between quality of life and burnout on athletes. Age 2014;24(32): 119.

6. King CR, Hinds PS. Quality of life: from nursing and patient perspectives: Jones \& Bartlett Publishers; 2011.

7. Si-Ying W, Huang-Yuan L, Jun T, Wei Z, Jian L, Xiao-Rong W. Health-related quality of life and its main related factors among nurses in China. Industrial health 2011;49(2): 158-65.

8. Ministry of Health. Vietnam Health Statistics Yearbook. 2014. 
9. Nguyen TTD. Understanding knowledge and care satisfaction of Da Nang Hospital. 2009.

10. Krejcie RV, Morgan DW. Determining sample size for research activities. Educational and psychological measurement 1970;30(3): 607-10.

11. Grant JS, Davis LL. Selection and use of content experts for instrument development. Research in nursing \& health 1997;20(3): 269-74.

12. Polit DF, Beck CT. Nursing research: Principles and methods: Lippincott Williams \& Wilkins; 2004.

13. Lahana E, Papadopoulou K, Roumeliotou O, Tsounis A, Sarafis P, Niakas D. Burnout among nurses working in social welfare centers for the disabled. BMC nursing 2017;16(1): 15.

14. Piri Y, Zeinali A. Relationship between Perceived Social Support, Social Capital and Quality of Life with Job Burnout among Nurses. Iran Journal of Nursing 2016;29(103): 13-23.

15. Aytekin A, Yilmaz F, Kuguoglu S. Burnout levels in neonatal intensive care nurses and its effects on their quality of life. Australian Journal of Advanced Nursing, The 2013;31(2): 39.

16. Kapucu SS, Akkuş Y, Akdemir N, Karacan Y. The burnout and exhaustion levels of nurses working in haemodialysis units. Journal of renal care 2009;35(3): 134-40.

17. Aiken LH, Clarke SP, Sloane DM, et al. Nurses' reports on hospital care in five countries. Health affairs 2001;20(3): 43-53.

18. Dehghan Nayeri N, Salehi T, Ali Asadi Noghabi A. Quality of work life and productivity among Iranian nurses. Contemporary nurse 2011;39(1): 106-18.

19. Delgoshyii B, Riahi L, Motaghi M. Relationship of quality of working life in Kashan teaching and nonteaching hospitals with knowledge management according to the top and middle manager's point of view. J Hostpital 2010;9: 67-74.

20. Mohammadi A, Sarhanggi F, Ebadi A, et al. Relationship between psychological problems and quality of work life of Intensive Care Unit Nurses. Iranian Journal of Critical Care Nursing (IJCCN) 2011;4(3): 135-40.

21. Ba Tham Ho. Some factors impact on the quality of life for Ho Chi Minh city residents in current context. Scientific Conference: Quality of life of Ho Chi Minh City people in the current economic context. 2012.

22. Milosevic M, Golubic R, Knezevic B, Golubic K, Bubas M, Mustajbegovic J. Work ability as a major determinant of clinical nurses' quality of life. Journal of clinical nursing 2011;20(19-20): 2931-38.

23. Shao MF, Chou YC, Yeh MY, Tzeng WC. Sleep quality and quality of life in female shift-working nurses. Journal of advanced nursing 2010;66(7): 1565-72.

24. YAZDI MH, Estaji Z, HEYDARI A. Study of the quality of life of nurses in Sabzevar hospitals in 20052006. 2009.

25. Honkonen $\mathrm{T}$, Ahola $\mathrm{K}$, Pertovaara $\mathrm{M}$, et al. The association between burnout and physical illness in the general population-results from the Finnish Health 2000 Study. Journal of psychosomatic research 2006;61(1): 59-66. 
26. Kim H, Ji J, Kao D. Burnout and physical health among social workers: A three-year longitudinal study. Social work 2011;56(3): 258-68. 